\title{
On Cartesian Nets and Fubini-Tonelli Type Theorem
}

\author{
Mangatiana A. Robdera* \\ Department of Mathematics, University of Botswana, 4775 Notwane Road, Gaborone, Botswana. \\ *Corresponding author. Tel.: +26775304025; email: robdera@yahoo.com \\ Manuscript submitted June 12, 2016; accepted November 4, 2016. \\ doi: 10.17706/ijapm.2017.7.2.118-127
}

\begin{abstract}
The notion of Cartesian net is introduced in order to provide an appropriate setting for the notion of double limit for directed functions defined on Cartesian products of spaces. As an application, we obtain extensions of the Fubini-Tonelli type theorem to the setting of the extended notion of integrability recently introduced.
\end{abstract}

Key words: Cartesian nets, Fubini-Tonelli theorem, vector integration.

\section{Introduction}

In the last half century, the study of the theory of vector valued integration has gained considerably in depth and in scope (see for example [1]-[6]). Recently, a relatively new approach to such a theory has been introduced in [1] and some important generalizations of the classical results have been proposed and studied in [7]-[10]. Such an approach strengthens the various existing classical concepts of integral and provides a continuous thread tying the subject matter together. The classical theorem of Fubini and Tonelli is another important result in the theory of integration. It proves to be very useful and oftentimes indispensable technical tools that provides conditions under which it is possible to compute a double integral using iterated integrals. However, the proof of such a theorem is always lengthy, too technical, and require certain level of abstraction. This is due mainly to the heaviness of the countable additivity condition for measures and the measurability requirement for functions in the statements of such a theorem.

In this paper, we are concerned with the formulation of the Fubini-Tonelli theorem in the setting of the extended integration theory for vector valued functions. Our approach will offer, not only generalizations, but also and more importantly provide significantly simpler, thus easily understood proofs. Our development is facilitated by the introduction of the notions of Cartesian net that will allow us to generalize the notion of double limit.

The exposition will be organized as follows. In Section 2, we generalize the notion of limit for double sequences to the case of nets. The results in this section are of importance in their own right and therefore can be seen as of independent interest. However, they will constitute the basics for the proofs of our main results that will be presented in the last section 4. A brief review of the essentials on the notion of the extended integral shall be presented in Section 3.

\section{Cartesian Nets}

In this section, we introduce the notion of Cartesian nets and extend the notion of double limit of sequences to the net limits. We essentially mimic the concept of double limit for sequences. We study the relationship between Cartesian limit and iterated limits for nets. 
Recall that a nonempty set $\Omega$ is said to be a directed set if a binary relation is defined on $\Omega$ with the following properties:

1) Transitivity: for $\omega, \gamma, \delta \in \Omega$, if $\omega>\gamma$ and $\gamma>\delta$ then $\omega>\delta$;

2) Upper-bound property: for $\omega, \gamma \in \Omega$, there exists $\delta \in \Omega$, such that $\delta>\gamma$ and $\delta>\omega$.

We then say that "the relation $>$ defines a direction on $\Omega$ " or that "the set $\Omega$ is directed by the relation $>$ ". Such a notion shall simply expressed by the giving of the pair $(\Omega,>)$. The relation $\omega>\gamma$ can be thought of, or read as " $\omega$ is after $\gamma$ ". A function defined on a directed set is customary called a net. More precisely, a net of elements of a set $X$ is a function defined on some directed set $(\Omega,>)$ taking values in $X$.

In the special case where $X$ is a topological space, one can talk about the notion of convergence. We say that a net $\phi:(\Omega,>) \rightarrow X$ converges to an element $a \in X$, if for every neighborhood $N$ of $a$, there exists $\omega_{0} \in \Omega$ such that $\phi(\omega) \in N$ whenever $\omega>\omega_{0}$. The element $a$ is then called the limit of the net $\phi$ along the direction, and we write $a=\lim _{(\Omega,>)} \phi$. For simplicity of notation, we shall omit such parts of the symbolism under 'lim' as can be without danger of confusion. For more details and expository presentation of the notion of net-limit, the reader is referred to [11].

Assume that $(\Omega,>)$ and $\left(\Omega^{\prime},>^{\prime}\right)$ are two directed sets. One can naturally define a direction $>x>^{\prime}$ on the Cartesian product $\Omega \times \Omega^{\prime}$ as follows: $\left(\omega, \omega^{\prime}\right)>\times>^{\prime}\left(\gamma, \gamma^{\prime}\right)$ if $\omega>\gamma$ and $\omega^{\prime}>\gamma^{\prime}$. Such a direction shall be called the Cartesian direction on and the directed set $\left(\Omega \times \Omega^{\prime},>\times>>^{\prime}\right)$ is going to be simply called the Cartesian product of the directed sets $(\Omega,>)$ and $\left(\Omega^{\prime},>^{\prime}\right)$. We shall call a Cartesian net a function defined on a Cartesian product of two directed sets.

Let $\phi:\left(\Omega \times \Omega^{\prime},>\times>^{\prime}\right) \rightarrow X$ be a Cartesian net taking value in a topological space $X$. For each

$\omega \in \Omega$, the function $\omega^{\prime} \mapsto \phi\left(\omega, \omega^{\prime}\right)$ is a net defined on $\left(\Omega^{\prime},>^{\prime}\right)$. In what follows, by " $\lim _{\left(\Omega^{\prime},>^{\prime}\right)} \phi$ exists" we mean for every $\omega \in \Omega, \lim _{\left(\Omega^{\prime}, \succ^{\prime}\right)} \phi\left(\omega,{ }^{\circ}\right)$ exists. Likewise, for each $\omega^{\prime} \in \Omega^{\prime}$, the function $\omega \mapsto \phi\left(\omega, \omega^{\prime}\right)$ is a net defined on $(\Omega,>)$ and by " $\lim _{(\Omega,>)} \phi$ exists" we mean for every $\omega^{\prime} \in \Omega^{\prime}, \lim _{(\Omega,>)} \phi\left(\cdot, \omega^{\prime}\right)$ exists.

For each Cartesian net, there correspond five important limits:

1) $\lim _{>x>^{\prime}} \phi$;

2) $\lim _{\left(\Omega^{\prime}, \succ^{\prime}\right)} \phi ; \lim _{(\Omega,>)} \phi$;

3) $\lim _{(\Omega,>)}\left(\lim _{\left(\Omega^{\prime},>^{\prime}\right)} \phi\right) ; \lim _{\left(\Omega^{\prime},>^{\prime}\right)}\left(\lim _{(\Omega,>)} \phi\right)$

The limit in 1. is called Cartesian limit, those in 2. are known as partial limits, whereas the limits in 3. shall be termed as iterated limits.

The following example shows that existence of the iterative limits does not imply the existence of the Cartesian limit.

Example 1. Let $\phi:(\mathbb{R} \times \mathbb{R},>\times>) \rightarrow \mathbb{R}$ be the net defined by $\phi(x, y)=\frac{x}{1+x+y}$, where $>\times>$ is the Cartesian direction of the two copies of $\mathbb{R}$, each directed towards $+\infty$. Then $\lim _{x \rightarrow \infty}\left(\lim _{y \rightarrow \infty} \phi(x, y)\right)=0$, $\lim _{y \rightarrow \infty}\left(\lim _{x \rightarrow \infty} \phi(x, y)\right)=1$, and $\lim _{>x>^{\prime}} \phi=\lim _{(x, y) \rightarrow(\infty, \infty)} \phi(x, y)$ does not exists.

Conversely, the existence of the Cartesian limit does not guarantee the existence of the iterative limits.

Example 2. Let $\phi:(\mathbb{N} \times \mathbb{N},>\times>) \rightarrow \mathbb{R}$ be the net defined by $\phi(n, m)=(-1)^{m}\left(\frac{1}{n}+\frac{1}{m}\right)$, where $>\times>$ is the Cartesian direction of the two copies of $\mathbb{N}$, each directed towards $+\infty$. Then $\lim _{m \rightarrow \infty}\left(\lim _{n \rightarrow \infty} \phi(n, m)\right)=0, \lim _{n \rightarrow \infty}\left(\lim _{m \rightarrow \infty} \phi(n, m)\right)$ does not exists, $\lim _{\succ x \succ^{\prime}} \phi=0$.

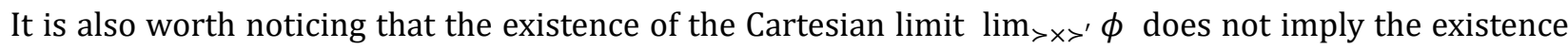
of the partial $\operatorname{limitslim}_{\left(\Omega^{\prime}, \succ^{\prime}\right)} \phi$ or $\lim _{(\Omega,>)} \phi$ as the following example shows.

Example 3. Let $\phi: \mathbb{N} \times \mathbb{N},>\times>) \rightarrow \mathbb{R}$ be the net defined by $\phi(n, m)=(-1)^{n}\left(\frac{1}{m}\right)$. Then the Cartesian limit $\lim _{\succ x \succ^{\prime}} \phi$ is 0 while $\lim _{n \rightarrow \infty} \phi(n, m)$ does not exist.

Our first result gives a necessary and sufficient conditions for the existence of the iterated limit of a convergent Cartesian net taking values in a metric space $(X, d)$. 
Theorem 1. Let $\phi:\left(\Omega \times \Omega^{\prime},>\times>^{\prime}\right) \rightarrow X$ be a Cartesian net taking value in a metric space $(X, d)$. Assume that Cartesian limit $\lim _{>x \succ^{\prime}} \phi$ exists and is equal to $a$ in $X$. Then

1) $\lim _{(\Omega,>)}\left(\lim _{\left(\Omega^{\prime},>^{\prime}\right)} \phi\right)$ exists and equals to $a$ if and only if $\lim _{\left(\Omega^{\prime}, \succ^{\prime}\right)} \phi$ exists.

2) $\lim _{\left(\Omega^{\prime}, \succ^{\prime}\right)}\left(\lim _{(\Omega,>)} \phi\right)$ exists and equals to $a$ if and only if $\lim _{(\Omega, \succ)} \phi$ exists.

Proof. By symmetry, we only need to show the equivalence in 1 . The necessity is obvious. For the

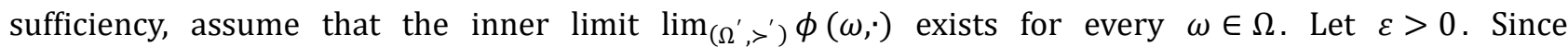
$\lim _{>x \succ^{\prime}} \phi=a$ there exists $\left(\omega_{0}, \omega_{0}^{\prime}\right) \in \Omega \times \Omega^{\prime}$ such that if $\omega>\omega_{0}$ and $\omega^{\prime}>\omega_{0}^{\prime}$ then $d\left(\phi\left(\omega, \omega^{\prime}\right), 0\right)<\frac{\varepsilon}{2}$.

Since for each $\omega \in \Omega, \lim _{\left(\Omega^{\prime},>^{\prime}\right)} \phi(\omega, \cdot):=b_{\omega}$ exists, there exists $\omega_{1}^{\prime} \in \Omega^{\prime}$ such that if $\omega^{\prime}>\omega_{1}^{\prime}$ then $d\left(\phi\left(\omega, \omega^{\prime}\right), b_{\omega}\right)<\frac{\varepsilon}{2}$. Choose $\omega_{2}^{\prime} \in \Omega^{\prime}$ such that $\omega_{2}^{\prime}>\omega_{0}^{\prime}, \omega^{\prime}{ }_{1}$. It follows that if $\omega>\omega_{0}$ and $\omega_{2}^{\prime}>\omega_{0}^{\prime}$ we have $d\left(a, b_{\omega}\right) \leq d\left(a, \phi\left(\omega, \omega^{\prime}\right)\right)+d\left(\phi\left(\omega, \omega^{\prime}\right), b_{\omega}\right) \leq \varepsilon$. This completes the proof.

As an immediate corollary, we have

Theorem 2. Let $\phi:\left(\Omega \times \Omega^{\prime},>\times>^{\prime}\right) \rightarrow X$ be a Cartesian net taking value in a metric space $(X, d)$. Assume that the Cartesian $\operatorname{limit}_{\lim _{>x \succ^{\prime}} \phi}$ exists and is equal to $a$ in $X$. Then the iterated limits $\lim _{(\Omega,>)}\left(\lim _{\left(\Omega^{\prime},>^{\prime}\right)} \phi\right)$ and $\lim _{\left(\Omega^{\prime},>^{\prime}\right)}\left(\lim _{(\Omega,>)} \phi\right)$ exist and both are equal to $a$ if and only if both partial $\operatorname{limitslim}_{\left(\Omega^{\prime}, \succ^{\prime}\right)} \phi$ and $\lim _{(\Omega,>)} \phi$ exist.

The next result can be considered as a partial converse of the previous theorem.

Theorem 3. Let $\phi:\left(\Omega \times \Omega^{\prime},>\times>^{\prime}\right) \rightarrow X$ be a Cartesian net taking values in a metric space $(X, d)$. Assume that

1) $\lim _{\left(\Omega^{\prime},>^{\prime}\right)}\left(\lim _{(\Omega,>)} \phi\right)$ exists and is equal to $a$ and

2) $\lim _{(\Omega,>)} \phi\left(\cdot, \omega^{\prime}\right)$ exists uniformly on $\omega^{\prime} \in \Omega^{\prime}$.

Then the Cartesian limit $\lim _{>x \succ^{\prime}} \phi$ exists and is equal to $a$.

Proof. By the second condition, the net $\omega \mapsto \phi\left(\omega, \omega^{\prime}\right)$ converges to $\omega \mapsto f\left(\omega^{\prime}\right):=\lim _{(\Omega, \succ)} \phi\left(\omega, \omega^{\prime}\right)$ uniformly on $\omega^{\prime} \in \Omega^{\prime}$. Given $\varepsilon>0$, there exists $\omega_{0} \in \Omega$ such that whenever $\omega>\omega_{0}$,

$$
\sup \left\{d\left(\phi\left(\omega, \omega^{\prime}\right), f\left(\omega^{\prime}\right)\right): \omega^{\prime} \in \Omega^{\prime}\right\}<\varepsilon / 2
$$

By the first condition, there exists $\omega_{0}^{\prime} \in \Omega^{\prime}$ such that whenever $\omega^{\prime}>\omega_{0}^{\prime}, d\left(f\left(\omega^{\prime}\right), a\right)<\varepsilon / 2$. It follows that if $\omega>\omega_{0}$ and $\omega^{\prime}>\omega_{0}^{\prime}$, we have $d\left(a, \phi\left(\omega, \omega^{\prime}\right)\right) \leq d\left(a, f\left(\omega^{\prime}\right)\right)+d\left(f\left(\omega^{\prime}\right), \phi\left(\omega, \omega^{\prime}\right)\right) \leq \varepsilon$. This completes the proof.

\section{Extended Notion of Integral}

The ordinary definition of the Lebesgue-Bochner integral has a straight forward generalization with the so-called vector valued McShane integral, but this generalization does not prove perfectly satisfactory. In this section, we recall the essential elements for the definition of the extended notion of integrability of vector valued functions as introduced in [1] and further developed in [10].

In what follows by an integral space we always mean a triplet $(\Omega, \Sigma, \mu)$ consisting of a nonempty set $\Omega$, a semiring $\Sigma$ and a scalar set function $\mu: 2^{\Omega} \rightarrow \mathbb{F}$, where $\mathbb{F}=\mathbb{R}$ or $\mathbb{C}$ satisfying

- $\mu(\varnothing)=0$;

- $|\mu(A)| \leq|\mu(B)|$ whenever $A \subset B$ in $\Sigma$ (monotone);

- $\left|\mu\left(\cup_{i \in I} A_{i}\right)\right| \leq \sum_{i \in I}\left|\mu\left(A_{i}\right)\right|$ for every finite set $I$ and $A_{i} \in \Sigma$ (subadditive).

Such a set function will simply be called an integrator.

Given an integral space $(\Omega, \Sigma, \mu)$ and a subset $A \in 2^{\Omega}$, a $\Sigma$-subpartition $P$ of $A$ is any finite collection $\left\{I_{i}: I_{i} \subset A, I_{i} \in \Sigma, i=1, \ldots, n\right\}$ with the following properties that $Q\left(I_{i}\right)<\infty$ for all $i \in\{1, \ldots, n\}, I_{i} \subset A$, $I_{i} \in \Sigma$, and $I_{i} \cap I_{j}=\emptyset$ whenever $i \neq j$. A $\Sigma$-subpartition $P=\left\{I_{i}: i=1, \ldots, n\right\}$ is said to be tagged if a point 
$t_{i} \in I_{i}$ is chosen for each $i \in\{1, \ldots, n\}$. We shall write $=\left\{\left(I_{i}, t_{i}\right): i=1, \ldots, n\right\}$ if we wish to specify the tagging points. We denote by $\Pi(A, \Sigma)$ the collection of all tagged $\Sigma$-subpartitions of the set $A$. The mesh or the norm of $P \in \Pi(A, \Sigma)$ is defined to be $\|P\|=\max \{\mu(I): I \in P\}$. We denote by $\sqcup P:=\bigcup_{I \in P} I$.

If $P, Q \in \Pi(A, \Sigma)$, we say that $Q$ is a refinement of $P$ and we write $Q>P$ if $\|Q\| \leq\|P\|$ and $\sqcup P \subset \sqcup Q$.

It is readily seen that the relation $>$ is transitive on $\Pi(A, \Sigma)$, and if $P, Q \in \Pi(A, \Sigma)$, then

$$
P \vee Q:=\{I \backslash \sqcup Q, I \cap J, J \backslash \sqcup P: I \in P, J \in Q\}
$$

and we have $P \vee Q>P, Q$. Thus the relation $>$ has the upper bound property on $\Pi(A, \Sigma)$. Therefore the set $\Pi(A, \Sigma)$ is directed by the binary relation $>$. It is worth noticing that the refinement does not depend on the choice of the tagging points.

Let $f: \Omega \rightarrow X$, where $(X,\|\|$.$) is either a real or a complex normed vector space. Given a$ $\Sigma$-subpartition $P \in \Pi(A, \Sigma)$, we define the $\Sigma, \mu$-Riemann sum of $f$ at $P$ to be the vector $f_{\mu}(P):=$ $\sum_{I \in P} \mu(I) f\left(t_{I}\right)$. We notice that the function $P \mapsto f_{\mu}(P)$ is an $X$-valued net defined on the directed set $(\Pi(A, \Sigma),>)$. Our extended notion of integrability is as follows:

Definition 4. Let $(X,\|\|$.$) Be a normed space. A function f: \Omega \rightarrow X$ is said to be $\Sigma$, $\mu$-integrable over a set $A \in \Sigma$, with $\Sigma$, $\mu$-integral $\int_{A} f d \mu$ if for every $\varepsilon>0$, there exists $P_{0} \in \Pi(A, \Sigma)$, such that for every $P \in$ $\Pi(A, \Sigma), P>P_{0}$ we have $\left\|\int_{A} f d \mu-f_{\mu}(P)\right\| \leq \varepsilon$.

We shall denote by $\mathcal{J}(A, \Sigma, \mu, X)$ the space of all $X$-valued $\Sigma$, $\mu$-integrable functions on a set $A \in 2^{\Omega}$. It is worth noticing that if $\mu(A)=0$ then $f_{\mu}(P)=0$ for all $P \in \Pi(A, \Sigma)$, and therefore $\int_{A} f d \mu=0$. Therefore if $\mu(\{\omega \in A: f(\omega) \neq g(\omega)\})=0$, then $\int_{A} f d \mu=\int_{A} g d \mu$. It is quickly seen that the relation $f \sim g$ if $\mu(\{\omega \in A: f(\omega) \neq g(\omega)\})=0$ is an equivalence relation on $\mathcal{J}(A, \Sigma, \mu, X)$. We shall denote

$$
I(A, \Sigma, \mu, X):=\mathcal{J}(A, \Sigma, \mu, X) / \sim .
$$

For $p \in(0, \infty)$, a function $f: \Omega \rightarrow X$ is said to be $\Sigma, \mu, p$-integrable if the scalar function $\omega \mapsto\|f(\omega)\|^{p}$ is $\Sigma, \mu$ - integrable. We shall denote by $\mathcal{J}^{p}(A, \Sigma, \mu, X)$ (resp. $I^{p}(A, \Sigma, \mu, X)$ the space of all (resp. classes of) $X$-valued $\Sigma, \mu, p$-integrable functions on a set $A \in 2^{\Omega}$. It is easy to see that $\mathcal{J}^{p}(A, \Sigma, \mu, X) \subset \mathcal{J}(A, \Sigma, \mu, X)$ (resp. and $I^{p}(A, \Sigma, \mu, X) \subset I(A, \Sigma, \mu, X)$.

In the particular case where $\Sigma$ is a $\sigma$-algebra containing the Borel sets of a set $\Omega$, and the integrator $\mu$ is the Lebesgue measure on $\Sigma$, the Bochner integrable functions are $\Sigma, \mu, 1$-integrable. An extended version of the Dvoretski-Rogers theorem proved in [6] shows that if $X$ is an infinite dimensional vector space, the space of all $X$-valued Bochner integrable functions is a proper subspace of all $\Sigma$, $\mu$-integrable functions.

It is worth noticing that although the integrator is a function that may be defined on the whole $2^{\Omega}$, the value of the $\Sigma$, $\mu$-integral $\int_{A} f d \mu$ depends only on values taken by the integrator on $\Sigma$. Another important feature of the above definition of the integral is also the fact that no measurability conditions are required. Hence, integration can be defined for non-measurable functions. Integration can also be defined even over non-measurable sets. In fact, the integral does not depend on any topological structure of the domain space. For the latest developments on the new approach to integration, the reader is referred to [7]-[9].

We finish this section with a useful characterization of integrability. Assume that $f: \Omega \rightarrow X$ is $\Sigma, \mu$ -integrable over a set $A \in 2^{\Omega}$. Fix $\varepsilon>0$ and let $P_{0} \in \Pi(A, \Sigma)$ be such that for every $Q \in \Pi(A, \Sigma), Q>$ $P_{0}$ we have $\left\|\int_{A} f d \mu-f_{\mu}(Q)\right\| \leq \varepsilon / 2$. Fix such a subpartition $Q$. Then we also have for every $P \in \Pi(A, \Sigma)$ that does not intersect $P_{0}$, we have $P \vee Q>P_{0}$, and therefore $\left\|\int_{A} f d \mu-f_{\mu}(P \cup Q)\right\| \leq \varepsilon / 2$. It follows that 


$$
\left\|f_{\mu}(P)\right\|=\left\|f_{\mu}(P)-f_{\mu}(P \cup Q)\right\| \leq\left\|\int_{A} f d \mu-f_{\mu}(Q)\right\|+\left\|\int_{A} f d \mu-f_{\mu}(P \cup Q)\right\|<\varepsilon .
$$

Thus we have the following proposition.

Proposition 5. If $\mathrm{f}: \Omega \rightarrow \mathrm{X}$ is $\Sigma$, $\mu$ - integrable over a set $A \in 2^{\Omega}$, then for every $\varepsilon>0$, there exists $P_{0} \in \Pi(A, \Sigma)$ such that for every $P \in \Pi(A, \Sigma)$ that does not intersect $\mathrm{P}_{0}$, we have $\left\|f_{\mu}(P)\right\|<\varepsilon$.

We introduce the following definition.

Definition 6. A function $\mathrm{f}: \Omega \rightarrow \mathrm{X}$ is said to satisfy the Cauchy criterion for integrability over a set $\mathrm{A}$ if for every $\varepsilon>0$, there exists $P_{0} \in \Pi(A, \Sigma)$ such that for every $P \in \Pi(A, \Sigma), P \cap P_{0}=\emptyset$, we have $\left\|f_{\mu}(P)\right\|<\varepsilon$.

For the special case where $X$ is a Banach space, the Cauchy criterion for integrability over $A$ is exactly the Cauchy condition for the net $P \mapsto f_{\mu}(P)$. The next theorem is immediate from the general well known fact that for nets taking values in a Banach space, the Cauchy net condition is equivalent to the net convergence.

Theorem 7. Let $\mathrm{X}$ be a Banach space. Then a function $\mathrm{f}: \Omega \rightarrow \mathrm{X}$ is $\Sigma, \mu$-integrable over a set $A \in 2^{\Omega}$ if and only if it satisfies the Cauchy criterion for integrability over the set $\mathrm{A}$.

Let $(\Omega, \Sigma, \mu)$ be an integral space and let $\phi: \Omega^{\prime} \rightarrow \Omega$ be an injective function. It is easily follows from the injective property of $\phi$ that for every subpartition $P$ of the set $\Omega$, the set $\phi^{-1}(P)=\left\{\phi^{-1}(I): I \in P\right\}$ is a subpartition of $\Omega^{\prime}$. We denote $\phi^{-1}(\Pi(\Omega, \Sigma)):=\left\{\phi^{-1}(P): P \in \Pi(\Omega, \Sigma)\right\}$.

We also notice that the set function $K \mapsto \mu(\phi(K))=: \phi^{-1}(\mu)(K)$ defines an integrator on $\phi^{-1}(\Sigma)=\{K \in$ $\left.2^{\Omega^{\prime}}: \phi(K) \in \Sigma\right\}$. Now, let $f: \Omega \rightarrow X$ be a function and consider the net of its Riemann sums $P \mapsto f_{\mu}(P)$ on $\Pi(\Omega, \Sigma)$. For every $R \in \phi^{-1}(\Pi(\Omega, \Sigma))$, we have

$$
f_{\mu} \circ \phi(R)=\sum_{K \in R} \mu(\phi(K)) f\left(t_{K}\right)=\sum_{K \in R} \phi^{-1}(\mu)(K) f\left(t_{K}\right)=(f \circ \phi)_{\phi^{-1}(\mu)}(R) .
$$

Thus the mapping $R \mapsto(f \circ \phi)_{\phi^{-1}(\mu)}(R)$ is the Riemann sums of the function $f \circ \phi$ with respect to the integrator $\phi^{-1}(\mu)$.

On the other hand, if we assume that the set $\Omega$ is directed by a relation $>$, then one can naturally define a direction on the set $\phi^{-1}(\Sigma)$ by the relation $\phi(>)$ defined by $R \phi(>) S$ if $\phi(R)>\phi(S)$. It then follows that every injective function $\phi: \Omega^{\prime} \rightarrow \Omega$ defines a subnet $R \mapsto(f \circ \phi)_{\phi^{-1}(\mu)}(R)$ of the net of Riemann sums $P \mapsto f_{\mu}(P)$. The principal uses of subnets come by way of the theorem that if a net converges, every subnet converges to the same limit (see for example [11]). This fact implies at once the following:

Proposition 8. Let $(X,\|\|$.$) be a normed vector space and let \mu: \Sigma \subset 2^{\Omega} \rightarrow \mathbb{F}$ be a scalar integrator. A function $\quad \mathrm{f}: \Omega \rightarrow \mathrm{X}$ is $\Sigma, \mu$-integrable over a subset $A$ of $\Omega$ if and only if for every injective function $\phi: \Gamma \rightarrow \Omega$, the composition $f \circ \phi: \Gamma \rightarrow X$ is $\phi^{-1}(\Sigma), \phi^{-1}(\mu)$-integrable over A, where $\phi^{-1}(\Sigma)=\left\{K \in 2^{\Omega^{\prime}}: \phi(K) \in\right.$ $\Sigma\}$ and $\phi^{-1}(\mu)(K)=\mu(\phi(K))$ for every $K \in \phi^{-1}(\Sigma)$.

\section{Extended Formulation of the Fubini-Tonnelli Theorem}

Let $(\Omega, \Sigma, \mu)$ and $\left(\Omega^{\prime}, \Sigma^{\prime}, \mu\right)$ be two integral spaces. The set $\Sigma \times \Sigma^{\prime}=\left\{A \times A^{\prime}: A \in \Sigma, \mathrm{A}^{\prime} \in \Sigma^{\prime}\right\}$ is a semiring of subsets of the Cartesian product $\Omega \times \Omega^{\prime}$. The elements of $\Sigma \times \Sigma^{\prime}$ are called rectangles. We shall call Cartesian product of the integrators $\mu$ and $\mu^{\prime}$ any integrator $\pi: \Omega \times \Omega^{\prime} \rightarrow \mathbb{F}$ satisfying $\pi\left(A \times A^{\prime}\right)=$ $\mu(A) \mu^{\prime}\left(A^{\prime}\right)$ for all $A \in \Sigma$ and all $A^{\prime} \in \Sigma^{\prime}$. Since in defining the integral over some subsets of $\Omega \times \Omega^{\prime}$, we only need the values taken by the integrator on the semiring $\Sigma \times \Sigma^{\prime}$, we shall use the notation $\mu \times \mu^{\prime}$ for any Cartesian integrator. We then call the integral space $\left(\Omega \times \Omega^{\prime}, \Sigma \times \Sigma^{\prime}, \mu \times \mu^{\prime}\right)$ the Cartesian integral space (product of the two integral spaces $(\Omega, \Sigma, \mu)$ and $\left(\Omega^{\prime}, \Sigma^{\prime}, \mu^{\prime}\right)$ ). 
In what follows, we shall denote by $\Pi\left(\Omega \times \Omega^{\prime}, \Sigma \times \Sigma^{\prime}\right)$ the collection of all rectangle subpartitions of the form $P=\left\{I \times J: I \in \Pi(\Omega, \Sigma), J \in \Pi\left(\Omega^{\prime}, \Sigma^{\prime}\right)\right\}$. Given $P \in \Pi\left(\Omega \times \Omega^{\prime}, \Sigma \times \Sigma^{\prime}\right)$, we denote $P_{\Omega}:=\{I: I \times J \in P\}$ and $P_{\Omega^{\prime}}:=\{J: I \times J \in P\}$. On the set $\Pi\left(\Omega \times \Omega^{\prime}, \Sigma \times \Sigma^{\prime}\right)$, we shall consider the Cartesian direction $>\times>>^{\prime}$, where $>$ is the refinement on $\Pi(\Omega, \Sigma)$ and $>^{\prime}$ is the refinement on $\Pi\left(\Omega^{\prime}, \Sigma^{\prime}\right)$.

Given a metric space $(X, d)$ and a function $f: A \times A^{\prime} \rightarrow X$ a function, the Cartesian-Riemann sum at a given rectangular subpartition $P=\left\{I \times J: I \in \Pi(A, \Sigma), J \in \Pi\left(A^{\prime}, \Sigma^{\prime}\right)\right\}$ is

$$
f_{\mu \times \mu^{\prime}}(P)=\sum_{I \times J \in P} \mu(I) \mu^{\prime}(J) f\left(t_{I}, s_{J}\right)
$$

If $f$ is $\Sigma \times \Sigma^{\prime}, \mu \times \mu^{\prime}$-integrable over $A \times A^{\prime}$, that is, if $\int_{A \times A^{\prime}} f d \mu \times \mu^{\prime}=\lim _{\succ \times \succ^{\prime}} \sum_{I \times J \in P} \mu(I) \mu^{\prime}(J) f\left(t_{I}, s_{J}\right)$ exists, then $\int_{A \times A^{\prime}} f d \mu \times \mu^{\prime}$ shall be called the Cartesian-integral of $f$ over $A \times A^{\prime}$.

On the other hand, each $\omega \in A$ gives rise to a function $f_{\omega}$ defined on $A^{\prime}$ by $f_{\omega}\left(\omega^{\prime}\right)=f\left(\omega, \omega^{\prime}\right)$. The $\Sigma^{\prime}, \mu^{\prime}$-Riemann sum of $f_{\omega}$ at $P_{A^{\prime}}:=\{J: I \times J \in P\}$ is given by $f_{\omega, \mu^{\prime}\left(P_{A^{\prime}}\right)}=\sum_{J \in P_{A^{\prime}}} \mu^{\prime}(J) f\left(\omega, s_{J}\right)$. If $f_{\omega}$ is $\Sigma^{\prime}, \mu^{\prime}$-integrable over $A^{\prime}$, then its $\Sigma^{\prime}, \mu^{\prime}$-integral is given by $\int_{A^{\prime}} f_{\omega} d \mu^{\prime}=\lim _{\succ^{\prime}} \sum_{J \in P_{A^{\prime}}} \mu^{\prime}(J) f\left(\omega, s_{J}\right)$.

Assume that for every $\omega \in A$, the function $f_{\omega}$ is $\Sigma^{\prime}, \mu^{\prime}$-integrable over $A^{\prime \prime}$. Then the relation $\omega \mapsto$ $\phi^{\mathrm{A}}(\omega):=\int_{A^{\prime}} f_{\omega} d \mu^{\prime}$ obviously defines a function $\phi^{A}: A \rightarrow X$. The $\Sigma, \mu$-Riemann sum of $\phi^{A}$ at $P_{A}$ is given by

$$
\phi_{\mu}^{A}\left(P_{A}\right)=\sum_{I \in P_{A}} \mu(I) \phi^{A}\left(t_{I}\right)=\sum_{I \in P_{A}} \mu(I) \int_{A^{\prime}} f_{\omega} d \mu^{\prime}
$$

If $\phi^{A}$ is $\Sigma, \mu$ - integrable over $A$, then its integral $\int_{A} \phi^{A} d \mu$ is denoted by $\int_{A}\left(\int_{A^{\prime}} f d \mu^{\prime}\right) d \mu$ and is given by

$$
\lim _{>} \sum_{I \in P_{A}} \mu(I)\left(\lim _{>} \sum_{J \in P_{A^{\prime}}} \mu^{\prime}(J) f\left(t_{I}, s_{J}\right)\right)=\lim _{>} \lim _{>} \sum_{I \in P_{A}} \sum_{J \in P_{A^{\prime}}} \mu(I) \mu^{\prime}(J) f\left(t_{I}, s_{J}\right)
$$

Likewise, if each $\omega^{\prime} \in A^{\prime}$ gives rise to a function $f_{\omega^{\prime}}$ defined by $f_{\omega^{\prime}}(\omega)=f\left(\omega, \omega^{\prime}\right)$ that is $\Sigma$, $\mu$-integrable over $A$, then its $\Sigma$, $\mu$-integral is given by $\int_{A} f_{\omega^{\prime}} d \mu=\lim _{\succ} \sum_{I \in P_{A}} \mu(I) f\left(t_{l}, \omega^{\prime}\right)$. Also if the function $\omega^{\prime} \mapsto \phi^{A^{\prime}}\left(\omega^{\prime}\right):=\int_{A} f_{\omega^{\prime}} d \mu$ is $\Sigma^{\prime}, \mu^{\prime}$ - integrable over $A^{\prime}$, then its $\Sigma^{\prime}, \mu^{\prime}$-integral $\int_{A^{\prime}} \phi^{A^{\prime}} d \mu^{\prime}$ is denoted by $\int_{A^{\prime}}\left(\int_{A} f d \mu\right) d \mu^{\prime}$ and is given by

$$
\lim _{>} \sum_{J \in P_{A^{\prime}}} \mu^{\prime}(J)\left(\lim _{>} \sum_{I \in P_{A}} \mu(I) f\left(t_{I}, s_{J}\right)\right)=\lim _{>} \lim _{>} \sum_{J \in P_{A^{\prime}}} \sum_{I \in P_{A}} \mu(I) \mu^{\prime}(J) f\left(t_{I}, s_{J}\right)
$$

The integrals $\int_{A}\left(\int_{A^{\prime}} f d \mu^{\prime}\right) d \mu$ and $\int_{A^{\prime}}\left(\int_{A} f d \mu\right) d \mu^{\prime}$ are called the iterative integrals of the function $\mathrm{f}$ over the rectangle $A \times A^{\prime}$.

Our first main result follows immediately from Theorem 2 . It gives necessary and sufficient conditions for the equality of the iterative integrals and the Cartesian integral of a Cartesian integrable function. The result clearly extends the special case of the Fubini-Tonelli Theorem.

Theorem 9. Let $(X, d)$ be a given metric space and $\mathrm{f}: \Omega \times \Omega^{\prime} \rightarrow \mathrm{X}$ a function that is Cartesian-integrable over a rectangle $A \times A^{\prime} \subset \Omega \times \Omega^{\prime}$. Then 


$$
\int_{A}\left(\int_{A^{\prime}} f d \mu^{\prime}\right) d \mu=\int_{A^{\prime}}\left(\int_{A} f d \mu\right) d \mu^{\prime}=\int_{A \times A^{\prime}} f d \mu \times \mu^{\prime}
$$

if and only if

1) for every $\omega \in A$, the function $\omega^{\prime} \mapsto f\left(\omega, \omega^{\prime}\right)$ is $\Sigma^{\prime}, \mu^{\prime}$-integrable over $A^{\prime}$, and

2) for every $\omega^{\prime} \in A^{\prime}$, the function $\omega \mapsto f\left(\omega, \omega^{\prime}\right)$ is $\Sigma$, $\mu$-integrable over $A$.

Example 4. Using the Abel convergence test, the double series $\sum_{n, m=1}^{\infty} \frac{(-1)^{n+m}}{n m}$ is quickly seen to be convergent. Its sum is in fact the integral in the extended sense of the function $(n, m) \mapsto \frac{(-1)^{n+m}}{n m}$ over $\mathbb{N} \times \mathbb{N}$ with respect to the integrator $\mu \times \mu$ where $\mu(A)=\left\{\begin{array}{l}1 \text { if } A \neq \emptyset \\ 0 \text { otherwise. }\end{array}\right.$ It follows from the theorem that $\sum_{n, m=1}^{\infty} \frac{(-1)^{n+m}}{n m}=\int_{\mathbb{N}}\left(\int_{\mathbb{N}} \frac{(-1)^{n+m}}{n m} d \mu(n)\right) d \mu(m)=(\ln 2)^{2}$. It is worth noticing that the function $(n, m) \mapsto$ $\frac{(-1)^{n+m}}{n m}$ is not absolutely $\mu \times \mu$-integrable, that is, the double series $\sum_{n, m=1}^{\infty} \frac{(-1)^{n+m}}{n m}$ is not absolutely convergent.

Example 5. By comparison with the summable function $(n, m) \mapsto \frac{1}{\left(n^{2}+1\right)\left(m^{2}+1\right)}$, it is easy to see that the function $(x, y) \mapsto \frac{1}{\left(x^{2}+1\right)\left(y^{2}+1\right)}$ is Cartesian-integrable with respect to the Lebesgue integrator over $[0, \infty) \times[0, \infty)$. We also notice that for each $y \in[0, \infty)$ (resp. $x \in[0, \infty)$ ) the function $x \mapsto \frac{1}{\left(x^{2}+1\right)\left(y^{2}+1\right)}$ (resp. $y \mapsto \frac{1}{\left(x^{2}+1\right)\left(y^{2}+1\right)}$ ) is integrable over $[0, \infty)$. It follows from the theorem that

$$
\int_{[0, \infty) \times[0, \infty)} \frac{1}{\left(x^{2}+1\right)\left(y^{2}+1\right)} d x \times d y=\int_{0}^{\infty}\left(\int_{0}^{\infty} \frac{1}{\left(x^{2}+1\right)\left(y^{2}+1\right)} d x\right) d y=\int_{0}^{\infty} \frac{\pi}{2\left(y^{2}+1\right)} d y=\frac{\pi^{2}}{4}
$$

The following result follows immediately from Theorem 3 .

Theorem 10. Let $(X, d)$ be a given metric space and $\mathrm{f}: \Omega \times \Omega^{\prime} \rightarrow \mathrm{X}$. Assume that

1) $\int_{A^{\prime}}\left(\int_{A} f d \mu\right) d \mu^{\prime}$ exists and

2) $\omega \mapsto f\left(\omega, \omega^{\prime}\right)$ is $\Sigma, \mu$-integrable over A uniformly in $\omega^{\prime} \in A^{\prime}$.

Then $\mathrm{f}$ is Cartesian integrable over the rectangle $A \times A^{\prime}$ and $\int_{A \times A^{\prime}} f d \mu \times \mu^{\prime}=\int_{A^{\prime}}\left(\int_{A} f d \mu\right) d \mu^{\prime}$.

Example 6. It is known that the function $x \mapsto \frac{\sin x}{x}$ is not Lebesgue-integrable on $[0, \infty)$. In fact, one has $\int_{0}^{\infty}\left|\frac{\sin x}{x}\right| d x=\infty$. On the other hand, we have $\int_{0}^{\infty} \frac{\sin x}{x} d x=\frac{\pi}{2}$ and $\int_{-\infty}^{\infty} \frac{1}{1+x^{2}} d x=\pi$ in the sense of the extended integral with respect to the Lebesgue measure respectively on $[0, \infty)$ and on $(-\infty, \infty)$. We have

$$
\int_{-\infty}^{\infty}\left(\int_{0}^{\infty} \frac{\sin \left(1+y^{2}\right) x}{\left(1+y^{2}\right) x} d x\right) d y=\frac{\pi}{2} \int_{-\infty}^{\infty} \frac{1}{1+y^{2}} d y=\frac{\pi^{2}}{2}
$$

and for every $y \in(-\infty, \infty)$

$$
\left|\int_{0}^{\infty} \frac{\sin \left(1+y^{2}\right) x}{\left(1+y^{2}\right) x} d x\right| \leq \frac{\pi}{2} \frac{1}{1+y^{2}} \leq \frac{\pi}{2}
$$

It follows from the above theorem that the function $(x, y) \mapsto \frac{\sin \left(1+y^{2}\right) x}{\left(1+y^{2}\right) x}$ is integrable in the extended sense over $(-\infty, \infty) \times[0, \infty)$ with respect to the integrator $d x \times d y$ and 


$$
\int_{(-\infty, \infty) \times[0, \infty)} \frac{\sin \left(1+y^{2}\right) x}{\left(1+y^{2}\right) x} d x \times d y=\int_{-\infty}^{\infty}\left(\int_{0}^{\infty} \frac{\sin \left(1+y^{2}\right) x}{\left(1+y^{2}\right) x} d x\right) d y=\frac{\pi^{2}}{2}
$$

Again, it is worth noticing that $(x, y) \mapsto \frac{\sin \left(1+y^{2}\right) x}{\left(1+y^{2}\right) x}$ is not Lebesgue-integrable over $(-\infty, \infty) \times[0, \infty)$.

The following rearrangement theorem constitutes the essentials for the proof of the extended Fubini -Tonelli theorem whose full statement is postponed for a moment.

Theorem 11. Let $X$ be a normed space. Let $(\Omega, \Sigma, \mu)$ and $\left(\Omega^{\prime}, \Sigma^{\prime}, \mu^{\prime}\right)$ be two integral spaces where $\mu$ and $\mu^{\prime}$ are nonnegative integrators. Let $\mathrm{f}: \Omega \times \Omega^{\prime} \rightarrow \mathrm{X}$. Then for every one-to-one function $\varphi: \Gamma \rightarrow \Omega \times \Omega^{\prime}$, one has

$$
\int_{\Gamma}\|f \circ \varphi\| d \varphi^{-1}\left(\mu \times \mu^{\prime}\right)=\int_{\Omega}\left(\int_{\Omega^{\prime}}\|f(s, t)\| d \mu^{\prime}(t)\right) d \mu(s)=\int_{\Omega^{\prime}}\left(\int_{\Omega}\|f(s, t)\| d \mu(s)\right) d \mu^{\prime}(t)
$$

where $\varphi^{-1}\left(\mu \times \mu^{\prime}\right)(K)=\mu \times \mu^{\prime}(\varphi(K))$ for every $K \in \varphi^{-1}\left(\Sigma \times \Sigma^{\prime}\right)$.

Proof. Let $0 \leq \alpha \leq \int_{\Gamma}\|f \circ \varphi\| d \varphi^{-1}\left(\mu \times \mu^{\prime}\right)$. Choose $R_{0} \in \Pi\left(\Gamma, \varphi^{-1}\left(\Sigma \times \Sigma^{\prime}\right)\right)$ such that

$$
\alpha<\sum_{K \in R_{0}}\left\|f \circ \varphi\left(t_{K}\right)\right\| \varphi^{-1}\left(\mu \times \mu^{\prime}\right)(K) .
$$

Choose $P_{0} \in \Pi(\Omega, \Sigma)$ and $Q_{0} \in \Pi\left(\Omega^{\prime}, \Sigma^{\prime}\right)$ such that $\varphi\left(R_{0}\right) \subset P_{0} \times Q_{0}$. Then we have

$$
\begin{aligned}
\alpha & <\sum_{K \in R_{0}}\left\|f \circ \varphi\left(t_{K}\right)\right\| \varphi^{-1}\left(\mu \times \mu^{\prime}\right)(K) \leq \sum_{I \in P_{0}}\left(\sum_{J \in Q_{0}}\left\|f\left(r_{I}, s_{J}\right)\right\| \mu^{\prime}(J)\right) \mu(I) \\
& \leq \sum_{I \in P_{0}}\left(\int_{\Omega^{\prime}}\left\|f\left(r_{I}, s\right)\right\| d \mu^{\prime}(s)\right) \mu(I) \leq \int_{\Omega}\left(\int_{\Omega^{\prime}}\|f(r, s)\| d \mu^{\prime}(s)\right) d \mu(r) .
\end{aligned}
$$

Since $\alpha$ is arbitrary, we have $\int_{\Gamma}\|f \circ \varphi\| d \varphi^{-1}\left(\mu \times \mu^{\prime}\right) \leq \int_{\Omega}\left(\int_{\Omega^{\prime}}\|f(r, s)\| d \mu^{\prime}(s)\right) d \mu(r)$.

For the reverse inequality, let $0 \leq \beta \leq \int_{\Omega}\left(\int_{\Omega^{\prime}}\|f(r, s)\| d \mu^{\prime}(s)\right) d \mu(r)$. Choose $P_{1} \in \Pi(\Omega, \Sigma)$ such that

$$
\beta \leq \sum_{I \in P_{1}}\left(\int_{\Omega^{\prime}}\left\|f\left(r_{I}, s\right)\right\| d \mu^{\prime}(s)\right) \mu(I)=\int_{\Omega^{\prime}} \sum_{I \in P_{1}}\left\|f\left(r_{I}, s\right)\right\| \mu(I) d \mu^{\prime}(s) .
$$

We pick $Q_{1} \in \Pi\left(\Omega^{\prime}, \Sigma^{\prime}\right) \quad$ such that $\beta<\sum_{J \in Q_{1}}\left(\sum_{I \in P_{1}}\left\|f\left(r_{I}, s_{J}\right)\right\| \mu(I)\right) \mu^{\prime}()$. Then we choose $R_{1} \in \Pi\left(\Gamma, \varphi^{-1}\left(\Sigma \times \Sigma^{\prime}\right)\right)$ such that $\varphi^{-1}\left(P_{1} \times Q_{1}\right) \subset R_{1}$. Then we have

$$
\beta<\sum_{J \in Q_{1}}\left(\sum_{I \in P_{1}}\left\|f\left(r_{I}, s_{J}\right)\right\| \mu(I)\right) \mu^{\prime}(J) \leq \sum_{K \in R_{1}}\left\|f \circ \varphi\left(t_{K}\right)\right\| \varphi^{-1}\left(\mu \times \mu^{\prime}\right)(K) \leq \int_{\Gamma}\|f \circ \varphi\| d \varphi^{-1}\left(\mu \times \mu^{\prime}\right) .
$$

Since $\beta$ is arbitrary, it follows that $\int_{\Omega}\left(\int_{\Omega^{\prime}}\|f(r, s)\| d \mu^{\prime}(s)\right) d \mu(r) \leq \int_{\Gamma}\|f \circ \varphi\| d \varphi^{-1}\left(\mu \times \mu^{\prime}\right)$. Thus we have established that $\int_{\Gamma}\|f \circ \varphi\| d \varphi^{-1}\left(\mu \times \mu^{\prime}\right)=\int_{\Omega}\left(\int_{\Omega^{\prime}}\|f(r, s)\| d \mu^{\prime}(s)\right) d \mu(r)$. In a similar fashion, we obtain $\int_{\Gamma}\|f \circ \varphi\| d \varphi^{-1}\left(\mu \times \mu^{\prime}\right)=\int_{\Omega^{\prime}}\left(\int_{\Omega}\|f(r, s)\| d \mu(r)\right) d \mu^{\prime}(s)$.

We are in a position to state our main result that will take over the classical Fubini-Tonelli theorem.

Theorem 12. Let $(X,\|\cdot\|)$ be a normed vector space, $(\Omega, \Sigma, \mu)$ and $\left(\Omega^{\prime}, \Sigma^{\prime}, \mu^{\prime}\right)$ two integral spaces where 
$\mu$ and $\mu^{\prime}$ are nonnegative integrators, and $\mathrm{f}: \Omega \times \Omega^{\prime} \rightarrow \mathrm{X}$ a function. If one of the integrals

$$
\int_{\Omega \times \Omega^{\prime}}\|f\| d\left(\mu \times \mu^{\prime}\right), \int_{\Omega^{\prime}}\left(\int_{\Omega}\|f\| d \mu\right) d \mu^{\prime}, \int_{\Omega}\left(\int_{\Omega^{\prime}}\|f\| d \mu^{\prime}\right) d \mu
$$

exists, then each one of the other integrals exists and for every injective function $\varphi: \Gamma \rightarrow \Omega \times \Omega^{\prime}$,

$$
\begin{gathered}
\int_{\Omega \times \Omega^{\prime}}\|f\| d\left(\mu \times \mu^{\prime}\right)=\int_{\Omega^{\prime}}\left(\int_{\Omega}\|f\| d \mu\right) d \mu^{\prime}=\int_{\Omega}\left(\int_{\Omega^{\prime}}\|f\| d \mu^{\prime}\right) d \mu=\int_{\Gamma}\|f \circ \varphi\| d \varphi^{-1}\left(\mu \times \mu^{\prime}\right) ; \\
\int_{\Omega \times \Omega^{\prime}} f d\left(\mu \times \mu^{\prime}\right)=\int_{\Omega^{\prime}}\left(\int_{\Omega} f d \mu\right) d \mu^{\prime}=\int_{\Omega}\left(\int_{\Omega^{\prime}} f d \mu^{\prime}\right) d \mu=\int_{\Gamma} f \circ \varphi d \varphi^{-1}\left(\mu \times \mu^{\prime}\right) .
\end{gathered}
$$

Let us notice that for $1 \leq p<\infty$, the space $I^{p}(A, \Sigma, \mu, X)$ of the classes of $p$-norm integrable functions is naturally normed by $\|f\|_{p}=\left(\int_{A}\|f\|^{p} d \mu\right)^{1 / p}$. It is easy to see that when $\Sigma$ is a $\sigma$-algebra and when the integrator is the Lebesgue measure then the Lebesgue-Bochner space $L^{1}(\Omega, \Sigma, \mu, X)$ is a closed linear subspace of $I^{1}(\Omega, \Sigma, \mu, X)$. Also if $X$ is a Banach space, then $I^{1}(\Omega, \Sigma, \mu, X)$ is a Banach space (see for example [6]). The following theorem gives an alternative formulation of the Fubini-Tonelli theorem.

Theorem 13. Let X be a normed space, $(\Omega, \Sigma, \mu)$ and $\left(\Omega^{\prime}, \Sigma^{\prime}, \mu^{\prime}\right)$ two integral spaces where $\mu$ and $\mu^{\prime}$ are nonnegative integrators. Then each one of the following spaces is isometrically isomorphic to the other:

$$
I^{1}\left(\Omega \times \Omega^{\prime}, \Sigma \times \Sigma^{\prime}, \mu \times \mu^{\prime}, X\right), \quad I^{1}\left(\Omega^{\prime}, \Sigma^{\prime}, \mu^{\prime}, I^{1}(\Omega, \Sigma, \mu, X)\right), \quad \text { and } I^{1}\left(\Omega, \Sigma, \mu, I^{1}\left(\Omega^{\prime}, \Sigma^{\prime}, \mu^{\prime}, X\right)\right) .
$$

We end this paper with an application: we prove the following theorem concerning the multiplication of independent integrable functions.

Theorem 14. Let $X$ be a normed space. Let $f \in I^{1}(\Omega, \Sigma, \mu, X)$ and $g \in I^{1}\left(\Omega^{\prime}, \Sigma^{\prime}, \mu^{\prime}, X\right)$. Define on $\Omega \times \Omega^{\prime}$ the function $(x, y) \mapsto h(x, y)=f(x) g(y)$. Then $h \in I^{1}\left(\Omega \times \Omega^{\prime}, \Sigma \times \Sigma^{\prime}, \mu \times \mu^{\prime}, X\right)$ and

$$
\int_{\Omega \times \Omega^{\prime}} h d \mu \times \mu^{\prime}=\left(\int_{\Omega} f d \mu\right)\left(\int_{\Omega^{\prime}} g d \mu^{\prime}\right)
$$

Proof. It suffices to notice that for every $P \times Q \in \Pi(\Omega, \Sigma) \times \Pi\left(\Omega^{\prime}, \Sigma^{\prime}\right)=\Pi\left(\Omega \times \Omega^{\prime}, \Sigma \times \Sigma^{\prime}\right)$, one has

$$
\sum_{J \in Q}\left(\sum_{I \in P}\left\|h\left(t_{I}, s_{J}\right)\right\| \mu(I)\right) \mu^{\prime}(J)=\left(\sum_{I \in P}\left\|f\left(t_{I}\right)\right\| \mu(I)\right)\left(\sum\left\|g\left(s_{J}\right)\right\| \mu^{\prime}(J)\right) \leq\left(\int_{\Omega} f d \mu\right)\left(\int_{\Omega^{\prime}} g d \mu^{\prime}\right)<\infty
$$

This implies that the iterative integral $\int_{\Omega^{\prime}}\left(\int_{\Omega}\|h\| d \mu\right) d \mu^{\prime}$ exists. Hence, the above Tonelli-Fubini Theorem applies and yields the desired equality.

\section{Conclusions}

This paper can be considered as a continuation of recent works by the author on new approach integration theory. We define the notion of Cartesian net in order to extend the idea of iterated limit. We then suggest significantly simpler formulations and proofs of some extended forms of the Fubini and Tonelli theorems in the more general setting of vector valued functions. The strength of such extensions is the fact that the considered functions are no longer required to be measurable but just merely that the two iterated integrals are well defined and exist. The author believes that the interest of this paper lies not only in the obtained extension theorems, but also in the light it sheds on the very foundation of the study of integration 
theory.

\section{References}

[1] Robdera, M. A. (2013). Unified approach to vector valued integration. International Journal of Functional Analysis, Operator Theory and Application, 5(2), 119-139.

[2] Bartle, R. G. (1956). A general bilinear vector integral. Studia Math, 15, 337-352.

[3] Vector measures. (1977). American Mathematical Society. Providence, R.I. USA: Diestel, J. and Uhl Jr., J.J.

[4] Vector integration and stochastic integration in Banach spaces. (2000). USA: Dinculeanu, N.

[5] Dobrakov, I. (1979). On integration in Banach spaces III. Czechoslovak Math. Journal, 59(3), 478-499.

[6] Rodriguez, J. (2006). On integration of vector functions with respect to vector measures. Czechoslovak Mathematical Journal, 56(131), 805-825.

[7] Robdera, M. A. (2014). Tensor integral: A comprehensive approach to the integration theory. British Journal of Mathematics \& Computer Science, 4(22), 3236-3244.

[8] Robdera, M. A. (2015,) On non-metric covering lemma and extended Lebesgue differentiation theorem. British Journal of Mathematics \& Computer Science, 8(3), 220-228.

[9] Robdera, M. A. (2015). A natural characterization of tensor integrability. BJMCS Science, 8(6), 458-469.

[10] Robdera, M. A., \& Kagiso, D. (2013). On the differentiability of vector valued additive set functions. APM, 3 , 653-659.

[11] McShane, E.J. (1952). Partial orderings and moore-smith limits. american math. Monthly, 59, 1-11.

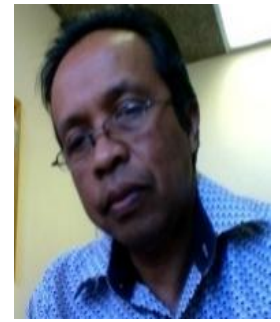

Mangatiana A. Robdera received his master's degree in pure mathematics from the University of Madagascar-Antananarivo in 1988, and his Ph.D. degree in pure mathematics at the University of Missouri-Columbia (USA) in 1996. He specializes in functional analysis.

Mangatiana has published several research papers at various renowned international peer reviewed journals such as Journal of Mathematical Analysis and Applications, Glasgow Journal of Mathematics, Quaestiones Mathematicae, Advanced in Pure Mathematics, International Journal of Modeling and Optimization. He also published two textbooks: A Concise Approach to Mathematical Analysis, (London, Springer-Verlag, 2003), A Concise Approach to Real Analysis, (www.lulu.com 2014). Mangatiana has worked in the area of Geometry of Banach spaces, Vector Measures, and Fixed Point Theory. Mangatiana currently has interests in works related to the theory of vector-valued integration and its applications in various areas of mathematical analysis.

Mangatiana has over twenty years of teaching experience at the tertiary education level at various institutions: University of Madagascar-Antananarivo, University of Missouri-Columbia (USA), William Paterson Univesrsity, New Jersey (USA), Eastern Mediterranean University, Northern Cyprus, Al-Akhawayn Univesity, Morocco. He currently holds an associate professor position at the University of Botswana in Gaborone.

Prof. Robdera is a member of the American Mathematical Society, the Southern African Mathematical Science Association, la Société de Mathématiques de Madagascar. In addition, Prof. Robdera is a member of the editorial board of the British Journal of Mathematics and Computer Science and avail himself as a reviewer for the American Mathematical Society. 